\title{
1995 CIF/IFC Gold Medal Winners
}

\section{Cégep de}

\section{I'Abitibi-Temiscamingue} Jasmin Caron

Notre récipiendaire se nomme Jasmin Caron. Originaire de Rouyn-Noranda, en Abitibi-Témiscamingue, Jasmin a su se démarquer de la moyenne académique du groupe tout au long de ses études en foresterie. Il a participé régulièrement aux différentes activités étudiantes du département et du collège. Parmi ses activités forestières courantes, on peut retenir entre autres la pratique de la chasse, la pêche et le vélo de montagne.

À travers ses études, Jasmin a occupé divers emplois qui l'ont amené graduellement à toucher le monde forestier. Il a d'ailleurs contribué à l'aménagement forestier de la forêt-école du Cégep en y réalisant divers travaux sylvicoles. Il a également contribuè à l'établissement de nouveaux sentiers d'interprétation au centre éducatif forestier de l'association forestière régionale. Jasmin a donc déjà laissé sa marque de forestier junior. Nous lui souhaitons donc une carrière remplie de défis.

\section{Laval \\ Vincent Fréchette}

Originaire de Québec, Vincent a terminé ses études secondaires à la polyvalente de Charlesbourg. Après une année en Technologie forestière à Rimouski, il est venu compléter ses deux dernières années à Sainte-Foy. Récipiendaire de la bourse REXFOR, Vincent s'est aussi mérité un Méritas

\section{Executive Director, continued from page 420}

The National Forest Week Reception was well attended by Members of Parliament and the forestry sector. The Honourable Anne McLellan presented CIF/IFC President Steve Tolnai with laminated copies of the Boreal Forest Map, and also announced the Maple Leaf as Canada's official arboreal emblem. The Honourable John Gerrard, announced the new Treevia program on the web in conjunction with the School Net program by Industry Canada. Tree Canada Foundation very kindly donated "Trees in a Sleeve" to the event and the Canadian Forest Service staff were out in full

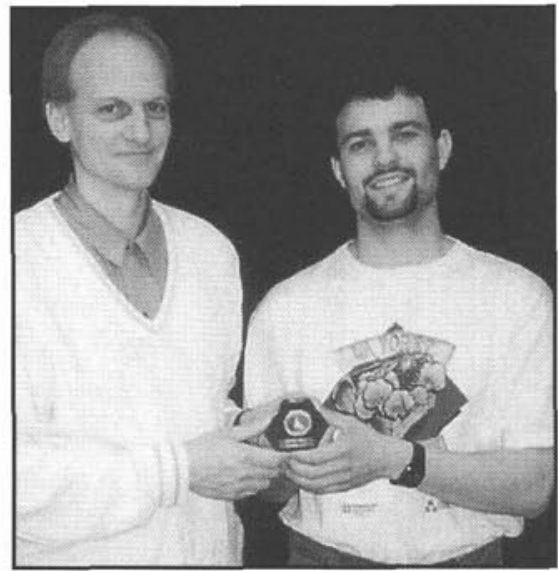

Jasmin Caron à droit avec André Roy.

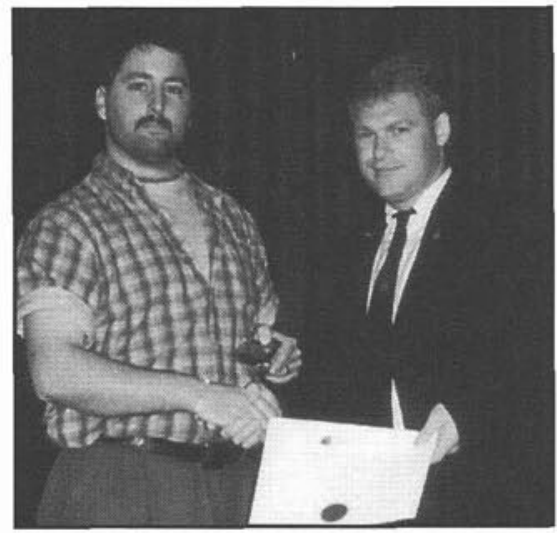

Bruce Jones

du Collège pour son engagement communautaire.

Adepte d'activités de plein air comme le canot, le camping et le trekking, il

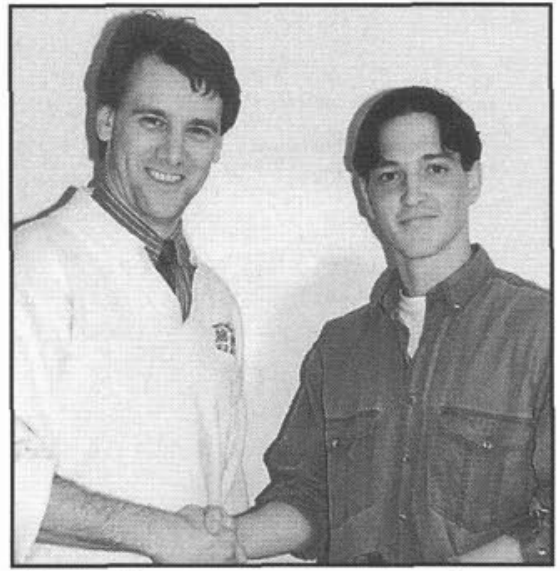

À gauche, J. P. Dansereau, à droit, V. Fréchette.

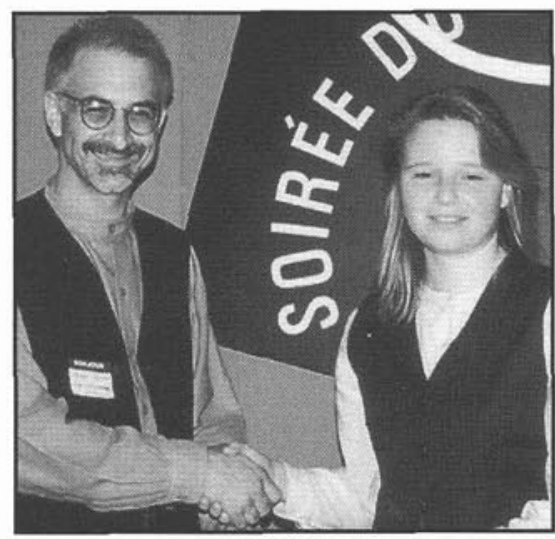

Michelle Caron s'adonne aussi dans ses moments libres à la musique.

Vincent souhaiterait faire carrière pour un organisme forestier oeuvrant en forêt habitée ou dans le domaine international. Ses performances scolaires et ses qualités le préparent sans aucun doute à un brillant avenir.

\section{Cégep de la Gaspésie et des îles Bruce Jones}

La médaille a été remise par monsieur Bruce Jones, enseignant en Aménagement forestier au Cégep de la Gaspésie et des îles. Un certificat d'excellence, de notre institution, accompagnait cette médaille lors de la cérémonie des Prix du Mérite Scolaire le 5 mai 1996.

\section{Cegep de Rimouski Michelle Caron}

C'est avec beaucoup de plaisir et d'intérêt que j'ai passé trois années à 
l'étude de l'aménagement forestier. Ayant grandi à l'intérieur d'une famille qui ne dépendait que du revenu de la ressource forestière, $\mathrm{j}$ 'ai rapidement compris toute l'importance de la protection et du maintien de la productivité de la forêt. Mes deux expériences de travail reliées à mon domaine d'étude ont été réalisées au sein du Ministère des Ressources Naturelles à Rivière-du-Loup et par la suite dans l'étourdissant mais palpitant monde du reboisement à Lebel-surQuévillon. Ces contacts avec la réalité quotidienne du travail de technologne en foresterie ont su concrétiser mon intérêt et surtout m'éveiller à la réalité de la foresterie.

Je suis une personne qui possède un respect considérable envers l'environnement et la faune. Je crois que le plus grand objectif de la foresterie devrait être la conciliation entre l'exploitation à grande échelle de la matière ligneuse, la conservation des habitats fauniques et, pardessus tout, le respect de la biodiversité. D'année en année, j'ai grand espoir qu'une nouvelle mentalité s'installera parmi tous ceux et celles qui oeuvrent en milieu forestier et que l'on parlera des «anciennes méthodes» quand on fera référence à certaines pratiques forestières d'aujourd'hui.

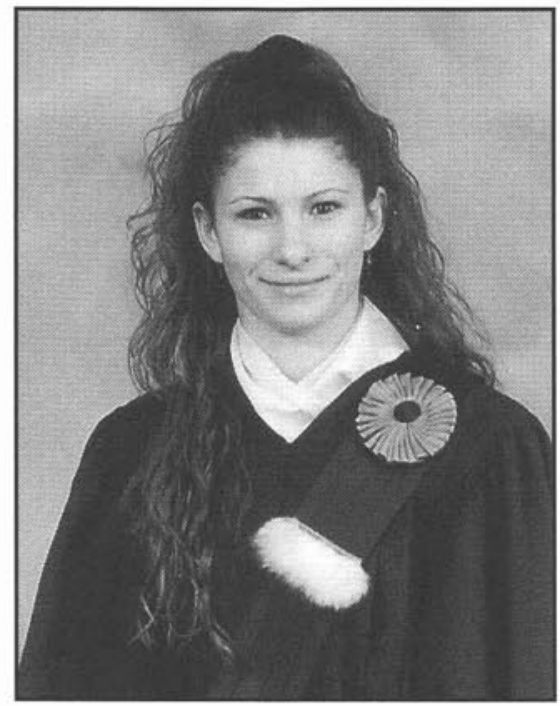

Nathalie Chartrand

\section{La Cité Collegiale, Hawksbury} Nathalie Chartrand

Nathalie Chartrand est originaire de Lefaivre (Ontario). Elle à fait des études à l'École secondaire de Plantagenet. Elle a reçu son diplôme d'étude secondaire ainsi que son attestation des cours pré-universitaire de l'Ontario en 1994. Nathalie s'inscrit en 1994 au programme de Techniques des sciences Forestières et

\section{The Forestry Chronicle Team}

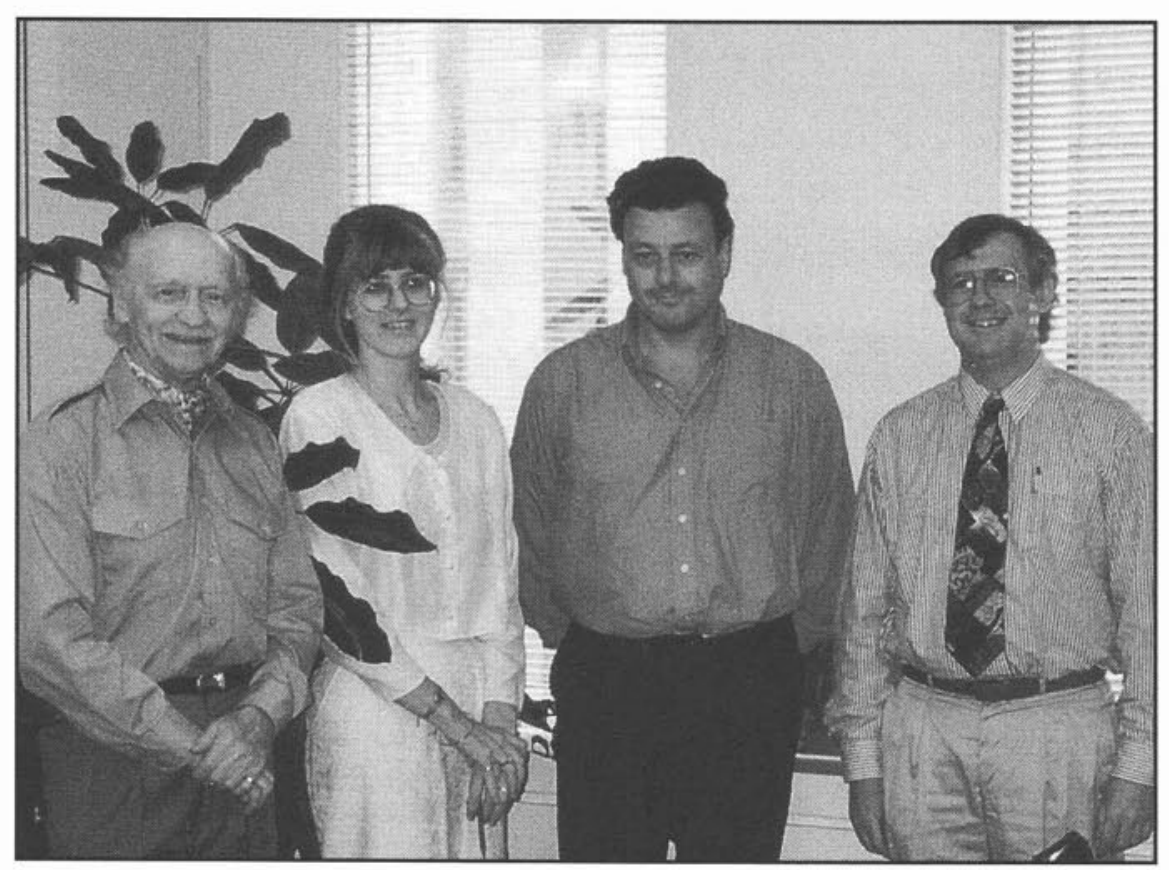

Fauniques car elle a un grand intérêt pour la faune et la flore. Pendant son séjour à La Cité collégiale de Hawkesbury elle se distingue par ses résultats académiques et sa vitalité.

Après l'obtention de son diplôme, Nathalie Chartrand décide de continuer ses études à l'université en écologie étant donné qu'elle veut contribuer de façon significative à la sauvegarde de l'environnement.

Elle attribut sa réussite scolaire au support de ses proches. Nathalie consacre ses moments libre à la lecture et à l'observation d'oiseaux.

Nathalie Chartrand graduated from high school in 1994. After reading the course description of the Techniques Forestières program at La Cité collégiale, she realized that this program was exactly what she wanted. She really enjoyed completing her program and her grades show it. Now that she has graduated at the college level she will continue on in ecology at university. She hopes the forest wildlife will benefit from her new knowledge and experience.

\section{British Columbia Institute of Technology Doug Reeve}

Forestry student Doug Reeve was thrilled to hear that he is being honored by the Canadian Institute of Forestry (CIF/IFC) for the outstanding scholarship, sports and citizenship he displayed during the program. Three months to graduation, Reeve can reflect upon the events that brought him to where he is today.

Prior to coming to BCIT, Reeve worked in the retail sector. When the company he worked for closed he seized the opportunity to go back to school. "I chose BCIT's Forestry program based on their excellent reputation and my love of being outdoors," says Reeve. "Prior to beginning the program I spent three summers working in Forest Health for the Ministry of Forests in Penticton. This confirmed my interest and I am looking forward to continuing on with my education."

Reeve hopes to complete a Forest Engineering degree at UBC and is particularly interested in forestry research.

Left: $L$ to $R$, Vidar Nordin, Co-Editor, Roxanne Comeau, CIF/IFC Executive Director, Tim Fenton, Production Manager, Darwin Burgess, Co-Editor. 